\title{
Finite-Time Terminal Sliding Mode Tracking Control for Piezoelectric Actuators
}

\author{
Jin $\mathrm{Li}^{1}$ and Liu Yang ${ }^{1,2}$ \\ ${ }^{1}$ College of Automation, Harbin Engineering University, Harbin, Heilongjiang 150001, China \\ ${ }^{2}$ Department of Earth and Space Science and Engineering, York University, Toronto, ON, Canada M3J 1P3
}

Correspondence should be addressed to Liu Yang; yangliuheu@gmail.com

Received 22 January 2014; Accepted 3 February 2014; Published 12 March 2014

Academic Editor: Shen Yin

Copyright (C) $2014 \mathrm{~J}$. Li and L. Yang. This is an open access article distributed under the Creative Commons Attribution License, which permits unrestricted use, distribution, and reproduction in any medium, provided the original work is properly cited.

This paper proposes a continuous finite-time control scheme using a new form of terminal sliding mode (TSM) combined with a sliding mode disturbance observer (SMDO). The proposed controller is applied for nanopositioning of piezoelectric actuators (PEAs). Nonlinearities, mainly hysteresis, can drastically degrade the system performance. Same as the model imperfection, hysteresis can also be treated as uncertainties of the system. These uncertainties can be addressed by terminal sliding mode control (TSMC) for it is promising for positioning and tracking control. To further improve the robustness of the TSM controller, the SMDO is employed to estimate the bounded disturbances and uncertainties. The robust stability of the TSMC is proved through a Lyapunov stability analysis. Simulation results demonstrate the effectiveness of the proposed TSM/SMDO controller for both positioning and tracking applications. The fast response, few chattering, and high precision positioning and tracking performances can be achieved in finite time by the proposed controller.

\section{Introduction}

Different from other traditional actuators, piezoelectric actuators (PEAs) possess the advantages of high positioning resolution, fast response, large actuating force, and free of backlash and friction [1]. Therefore, PEAs have been widely used in a variety of applications, such as adaptive optics [2], scanning tunneling microscopy $[3,4]$, data storage $[1,5,6]$, and nanofabrication. However, there are also some challenges in the use of PEAs. The main problems come from the nonlinear behaviors like creep and hysteresis that often occur when the PEAs are driven by an amplifier. These nonlinearities can greatly degrade the performance of PEAs and even compromise the stability of the closed-loop system $[2,7]$. For these two types of nonlinearity, creep is a slow drifting behavior in the displacement of PEAs, when responding to a step command voltage. Creep can cause a drifting steady state error in static or slow moving applications, but this effect can be easily eliminated by feedback techniques.

Hysteresis, on the other hand, is another typical nonlinear behavior that needs to be tackled in applications of PEAs. The hysteresis relation between the input voltage and the output displacement can cause normally $10 \%-15 \%$ of openloop positioning error in the displacement range of PEAs. Figure 1 shows the simulated hysteresis response of the PEA model employed in this research [8]. This phenomenon can largely degrade the performance of controllers that have not considered its influence. Some earlier works dealt with this problem by using charge amplifier [1,9-11] or restricting the amplitude of the input voltage small enough [12]. However, these two methods were either too complex or not practical in implementation. Therefore, researchers start to employ advanced control methods to suppress hysteresis in various applications of PEAs.

Past research proposed various control methods to deal with the influence of hysteresis. To generally summarize, mainly two ways of control strategies were employed in related literature. One way is using some inverse-based feedforward compensation methods, and another is using feedback control methods. In feedforward based methods, different hysteresis models are used to compensate this effect inversely. Typical models are Prandt-Ishlinskii model [13, 14], Preisach model [15, 16], Bouc-Wen model [17], and 


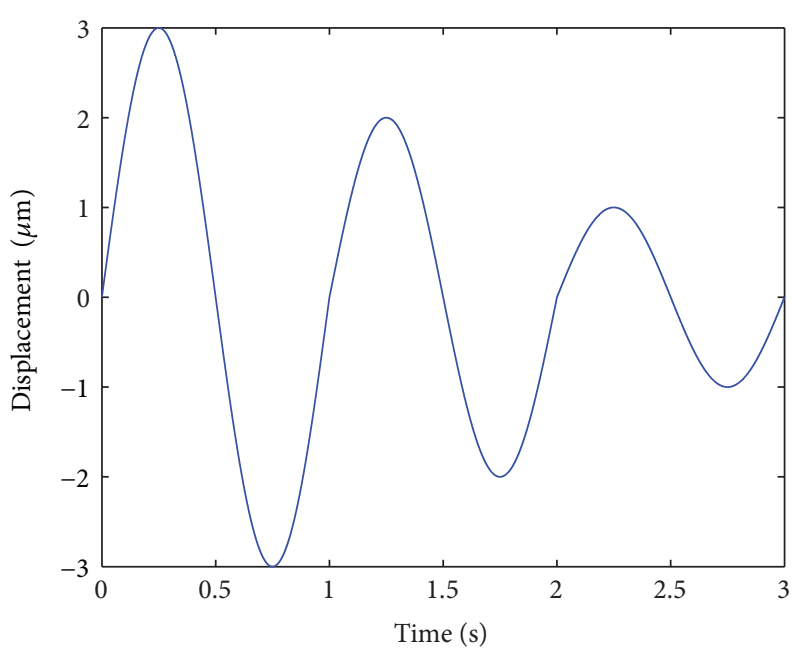

(a)

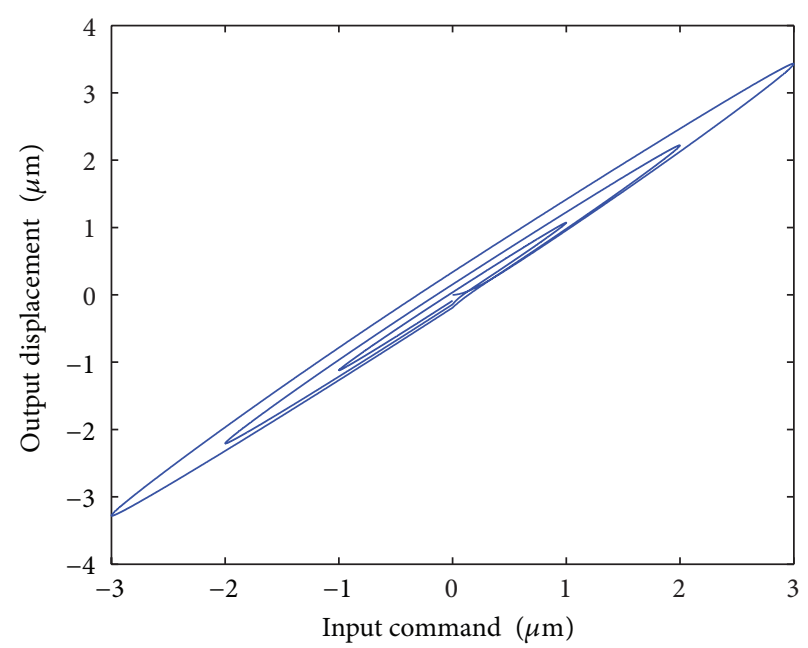

(b)

FIgURE 1: (a) A $1 \mathrm{~Hz}$ input displacement signal applied to the PEA model and (b) hysteresis loop obtained by simulation.

Maxwell resistive capacitor (MRC) model [18, 19]. However, these methods are based on precise hysteresis model; degraded compensation performance is unavoidable if modeling error exists. On the other hand, in feedback control methods, the hysteresis model is usually not needed since nonlinearities can be treated as disturbances that can be suppressed by feedback controller, related methods include PID (proportional-integral-derivative) control [20], repetitive control [21], robust control [22-26], and SMC (sliding mode control) [8, 27-29]. In addition, [30,31] combined those two types of methods by employing both feedforward and feedback control design.

It is well known that finite-time stabilization of dynamical systems will improve the systems performance of highprecision and finite-time convergence to the equilibrium. Therefore, discontinuous terminal sliding mode control with robustness for matched disturbances and parametric uncertainties with known bounds has been widely adopted in nonlinear systems for finite-time stability [32-35]. However, because of the chattering of discontinuous control, it may induce poor tracking performance and create undesirable oscillations in the control signal and even may excite highfrequency dynamics neglected in the course of modeling [8]. In order to alleviate chattering, the boundary layer technique is usually adopted. However, both the attractive SMC feature of insensitivity to uncertainties and disturbances and the finite time stability are lost. Recently, a continuous TSMC scheme has been developed for robotic manipulators to avoid this problem [36]. In this paper, a new continuous finite-time terminal sliding mode control combined with a sliding mode disturbance observer is proposed, which is then applied in a piezoelectric actuator system with finite-time stability. To improve the robustness of the TSMC, the SMDO is adopted to estimate the bounded disturbances and uncertainties in finite time. Here, the PEA is considered as a second-order nonlinear system to design the proposed controller, and the hysteresis considered as the main nonlinearity is modeled for accurate simulation. The stability of the proposed controller is proved by using the Lyapunov stability theory, and the positioning and tracking performances of the resulting control system illustrate that the proposed controller can provide the fast convergence in finite time and high tracking precision.

This paper is organized as follows. In Section 2, the problem formulation is presented. In Section 3, the continuous finite-time terminal sliding mode control with sliding mode disturbance observer scheme is designed. Simulations demonstration of the proposed controller is shown in Section 4 . Section 5 concludes this paper.

\section{Problem Formulation}

A class of second-order single input nonlinear systems with dynamic processes can be defined as follows:

$$
\ddot{x}=f(x, \dot{x})+b(x) u+f_{d},
$$

where $x$ and $\dot{x}$ are the system state variables, $f(x, \dot{x})$ is in general nonlinear and possibly time-varying, $b(x)$ expresses the control gain, $u$ is the control input, and $f_{d}$ represents the bounded external disturbance with $\left|f_{d}\right| \leq d$. $f(x, \dot{x})=$ $f_{n}(x, \dot{x})+\Delta f(x, \dot{x})$, and $b(x)=b_{n}(x)+\Delta b(x)$. Here $f_{n}(x, \dot{x})$ and $b_{n}(x)$ are the nominal parts, whereas $\Delta f(x, \dot{x})$ and $\Delta b(x)$ represent the perturbations in the system. Then, the secondorder system can be rewritten as

$$
\ddot{x}=f_{n}(x, \dot{x})+b_{n}(x) u+F_{d},
$$

where $f_{n}(x, \dot{x}), b_{n}(x)$ are the nominal parts and $F_{d}=$ $\Delta f(x, \dot{x})+\Delta b(x) u+f_{d}$ is the lumped system uncertainty, which is assumed to be bounded by $\left|F_{d}\right| \leq D$. $D$ is a given positive constant.

Consider the piezoelectric actuator as a second-order system [37], which can be written as

$$
\ddot{x}+2 \xi \omega_{n} \dot{x}+\omega_{n}^{2} x=k \omega_{n}^{2} u+F_{d},
$$

where $\xi, \omega_{n}$, and $k$ are the damping ratio, the natural frequency, and the gain of the second-order system, respectively. 


\section{Controller Design}

To have a concise manner of representation, in the rest of this paper, the system state variables $x$ and $\dot{x}$ will be omitted.

3.1. Terminal Sliding Mode Controller Design. For simplicity of expression and used in the analysis and design of the TSM controller, the following notion, which was used in [38], is introduced in this paper:

$$
\operatorname{sig}(x)^{\lambda}=|x|^{\lambda} \operatorname{sign}(x),
$$

where $0.5<\lambda<1$.

Remark 1. A TSM and a fast TSM can be described by the following first-order nonlinear differential equations [36]:

$$
\begin{gathered}
s=\dot{x}+\mu \operatorname{sig}(x)^{\lambda}=0, \\
s=\dot{x}+a x+\mu \operatorname{sig}(x)^{\lambda}=0,
\end{gathered}
$$

respectively, where $x \in R, a, \mu>0,0.5<\lambda<1$.

Remark 2. According to the definition of finite-time stability [39], the equilibrium point $x=0$ of the differential equations (5) and (6) is globally finite-time stable; for example, for any given initial condition $x(0)=x_{0}$, the system state $x$ will converge to 0 in finite time as follows:

$$
\begin{gathered}
T=\frac{1}{\mu(1-\lambda)}\left|x_{0}\right|^{(1-\lambda)}, \\
T=\frac{1}{a(1-\lambda)} \ln \frac{a\left|x_{0}\right|^{(1-\lambda)}+\mu}{\mu},
\end{gathered}
$$

respectively, and it stays there forever, such as $x=0$ for $t>T$. Define the tracking error as

$$
e_{0}=x-x_{d}
$$

where $x_{d}$ represents the desired position trajectory, and for the tracking task to be achievable using a feedback control $u$, the actuator output $x$ tracks the desired trajectory $x_{d}$ in finite time.

Introduce three auxiliary variables $e_{01}, e_{02}$, and $e$, where $\dot{e}_{01}=e_{0}, \dot{e}_{02}=e_{01}$, and

$$
e=\dot{e}_{01}+k_{0} e_{02}
$$

where $k_{0}$ is a positive constant.

Hence, a TSM sliding surface is defined as

$$
s=\dot{e}+\mu \operatorname{sig}(e)^{\lambda}
$$

where $\mu>0$ and $0.5<\lambda<1$. A continuous fast TSMtype reaching law is selected to achieve continuous control as follows:

$$
\dot{s}=-k_{1} s-k_{2} \operatorname{sig}(s)^{\rho},
$$

where $k_{1}, k_{2}>0$ and $0<\rho<1$.
By differentiating the sliding variable $s$ with respect to time, we have

$$
\dot{s}=-2 \xi \omega_{n} \dot{x}-\omega_{n}^{2} x+k \omega_{n}^{2} u+F_{d}-\ddot{x}_{d}+\mu \lambda|e|^{\lambda-1} \dot{e} .
$$

Substituting (11) into (12), the control law of the finitetime TSM controller can be obtained as follows:

$$
u=B^{-1}\left[-A-k_{1} s-k_{2} \operatorname{sig}(s)^{\rho}-F_{d}\right],
$$

where $A=-2 \xi \omega_{n} \dot{x}-\omega_{n}^{2} x+\mu \lambda|e|^{\lambda-1} \dot{e}-\ddot{x}_{d}$ and $B=k \omega_{n}^{2}$.

It can be seen from the expression equation (12) that the term $|e|^{\lambda-1} \dot{e}$ is included in the control law $u$ which has the negative fractional power $\lambda-1$ because of $0.5<\lambda<1$. Therefore, singularity will occur as $e=0$ and $\dot{e} \neq 0$. To avoid the singularly problem, the approach proposed in [40] is used in this paper. Define a new auxiliary variable $\bar{e}$ to replace the original $e$, which is written as

$$
\bar{e}= \begin{cases}|e|^{\lambda-1} \dot{e} & \text { if } e \neq 0 \text { and } \dot{e} \neq 0 \\ |\Delta|^{\lambda-1} \dot{e} & \text { if } e=0 \text { and } \dot{e} \neq 0 \\ 0 & \text { if } e=0 \text { and } \dot{e}=0\end{cases}
$$

where $\Delta>0$ is a small positive constant.

It should be noted that the bounded system uncertainty $F_{d}$ is always unknown and not available in general. Therefore, in order to increase the robustness of the controller and improve the control performance, a sliding mode disturbance observer is incorporated to estimate the uncertain terms.

3.2. Sliding Mode Disturbance Observer. The SMDO is designed as an effective way to improve the robustness to external disturbances and modeling uncertainties which can finish the estimation in finite time [41, 42]. To design a SMDO for estimating the bounded system uncertainty $F_{d}$, an auxiliary system is introduced as

$$
\begin{gathered}
\sigma=s+z, \\
\dot{z}=-A-B u-v,
\end{gathered}
$$

where $\sigma$ and $z$ are the auxiliary sliding variable and intermediate variable, respectively. $v$ is the auxiliary traditional SMC.

The $\sigma$ dynamic is derived, differentiating it with respect to time, we have

$$
\dot{\sigma}=\dot{s}+\dot{z}=F_{d}-v .
$$

Then the auxiliary traditional sliding mode control $v$ is designed to stabilize the sliding variable $\sigma$ at zero in finite time as follows:

$$
v=(D+\epsilon) \operatorname{sign}(\sigma),
$$

where $\epsilon>0$. Introduce a Lyapunov function $V=(1 / 2) \sigma^{2}$ to drive $\sigma$ to zero in finite time, and then compute its differentiating, we have

$$
V=\sigma \dot{\sigma}=\sigma\left(F_{d}-v\right) \leq|\sigma| D-|\sigma|(D+\epsilon)=-\epsilon|\sigma| .
$$

It can be conclude by using (17) that $\sigma$ converges to zero in finite time $t_{f}[41]$, which is

$$
t_{f} \leq \frac{|\sigma(0)|}{\epsilon}
$$


Therefore, the auxiliary system dynamics can be governed by equivalent control $v_{\mathrm{eq}} \cdot v_{\mathrm{eq}}$ is obtained by filtering the highfrequency switching control $v$ using a low pass filter, which is

$$
v_{\mathrm{eq}}=\frac{1}{\tau s+1} v,
$$

where $\tau>0$. For any $t$ satisfied $t>t_{f}$, the system uncertain term $F_{d}$ is estimated by $v_{\text {eq }}$ in finite time $t_{f}$, which is written as

$$
\widehat{F}_{d}=v_{\mathrm{eq}}
$$

where $\widehat{F}_{d}$ is the estimation of $F_{d}$. Then the final continuous TSM control law with SMDO is designed as

$$
u=B^{-1}\left[-A-k_{1} s-k_{2} \operatorname{sig}(s)^{\rho}-\widehat{F}_{d}\right] .
$$

Remark 3. The convergence of the auxiliary sliding variable $\sigma$ must be faster than that of $s$ to make sure that the terminal sliding variable is stabilized to zero only after the system uncertainty is estimated.

\subsection{Stability Analysis}

Lemma 4. Suppose that $c_{1}, c_{2}, \ldots, c_{n}$ and $0<p<2$ are all positive numbers; then the following inequality holds:

$$
\left(c_{1}^{2}+c_{2}^{2}+\cdots+c_{n}^{2}\right)^{p} \leq\left(c_{1}^{p}+c_{2}^{p}+\cdots+c_{n}^{p}\right)^{2} .
$$

Lemma 5. An extended Lyapunov description of finite-time stability can be given with the form of fast TSM equation (6) as [36]

$$
\dot{V}(x)+a V(x)+\mu V^{\lambda}(x) \leq 0,
$$

and the settling time can be given by

$$
T \leq \frac{1}{a(1-\lambda)} \ln \frac{a V^{1-\lambda}\left(x_{0}\right)+\mu}{\mu} .
$$

It is evident that the inequalities (24) and (25) mean exponential stability as well as faster finite-time stability.

Theorem 6. For a single-input second-order nonlinear system given by (3), with the terminal sliding surface defined by (10) and the reaching law given by (11), both the system robust stability and tracking convergence are guaranteed in finite time if the control law is designed as (22) based on the combination of SMDO.

Proof. Consider the following positive definite Lyapunov function:

$$
V=\frac{1}{2} s^{2}
$$

By taking the time derivative of $V$ with respect to time, we have

$$
\begin{aligned}
\dot{V} & =s \dot{s} \\
& =s\left(A+F_{d}+B u\right)
\end{aligned}
$$

$$
\begin{aligned}
& =s\left\{A+F_{d}+B\left[B^{-1}\left(-A-k_{1} s-k_{2} \operatorname{sig}(s)^{\rho}-\widehat{F}_{d}\right)\right]\right\} \\
& =-k_{1} s^{2}-s k_{2} \operatorname{sig}(s)^{\rho}+s \widetilde{F_{d}},
\end{aligned}
$$

where $A=-2 \xi \omega_{n} \dot{x}-\omega_{n}^{2} x+\mu \lambda|\bar{e}|^{\lambda-1} \dot{\bar{e}}-\ddot{x}_{d} \cdot \widetilde{F_{d}}=F_{d}-\widehat{F}_{d}$ since the sliding variable $s$ converges to zero only after the system uncertainty $F_{d}$ is estimated in finite time $t_{f}$. Thus, $\widetilde{F_{d}}=$ $F_{d}-\widehat{F}_{d} \rightarrow 0$, if $t>t_{f}$.

Therefore, for any $t>t_{f}$, from Lemma 4 , we have

$$
\dot{V} \leq-2 k_{1} V-2^{(\rho+1) / 2} k_{2} V^{(\rho+1) / 2},
$$

where $1 / 2<\rho<1$. According to Lemma 5, the proposed terminal sliding surface equation (10) will be reached in the finite time as follows:

$$
T \leq \frac{1}{k_{1}(1-\rho)} \ln \frac{k_{1} V^{(1-\rho) / 2}+2^{(\rho-1) / 2} k_{2}}{2^{(\rho-1) / 2} k_{2}} .
$$

Thus, according to the definition of (8), (9), and (10), if $s \rightarrow$ 0 in finite time $T$, then $e \rightarrow 0$ and $\dot{e} \rightarrow 0$ in finite time $T$, and then $e_{0} \rightarrow 0$ and $\dot{e}_{0} \rightarrow 0$ in finite time $T$; hence, $x \rightarrow x_{d}$ and $\dot{x} \rightarrow \dot{x}_{d}$ in finite time $T$. This shows that the proposed TSM controller combined with the SMDO ensures both the robust stability of the system and the convergence of the motion tracking.

\section{Simulation Results}

In this section, the proposed TSM controller combined with SMDO is validated through simulations. The results are shown and discussed in this section.

4.1. PEA Model. For the purpose of simulation, a BoucWen model which can describe the hysteresis is applied in this work. Consider the fact that the hysteresis is the major nonlinearity which can be handled as the uncertainty of the PEAs system. Thus, the hysteresis is modeled and integrated into the second-order PEA model for exact simulation. The Bouc-Wen model has already been verified that it is adaptive to describe the hysteresis loop of PEAs [43]. The piezoelectric actuator model with nonlinear hysteresis for simulation can be written as

$$
\begin{aligned}
& \ddot{x}+2 \xi \omega_{n} \dot{x}+\omega_{n}^{2} x=\omega_{n}^{2}(K u-h), \\
& \dot{h}=\alpha d \dot{u}-\beta|\dot{u}| h|h|^{n-1}-\gamma \dot{u}|h|^{n},
\end{aligned}
$$

where $h$ is the nonlinear hysteresis which indicates the hysteretic loop in terms of displacement whose magnitude and shape are determined by parameters $\alpha, \beta, \gamma$, the parameter $d$ is the piezoelectric coefficient, $u$ denotes the input voltage, and the order $n$ governs the smoothness of the transition from elastic to plastic response. For the elastic structure and material, $n=1$ is assigned in (31) as usual. These parameters used in this paper are from [8] and the values of these parameters are shown in Table 1. 


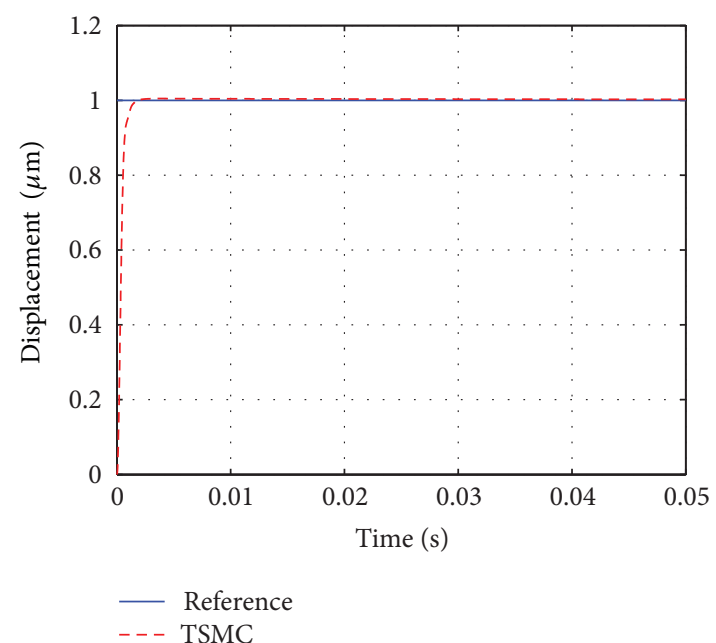

(a)

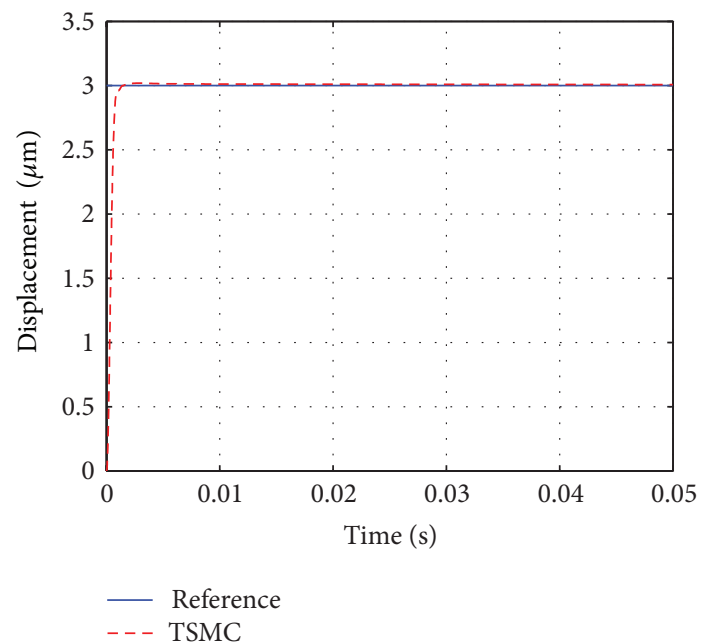

(c)

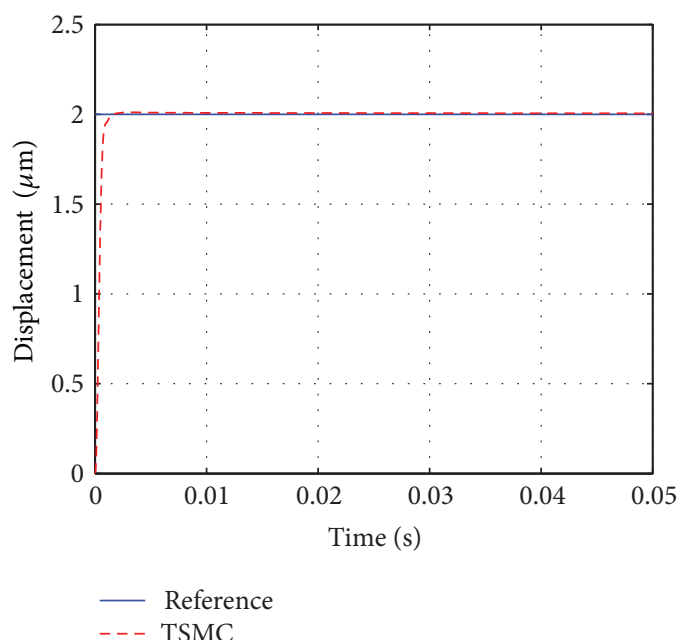

(b)

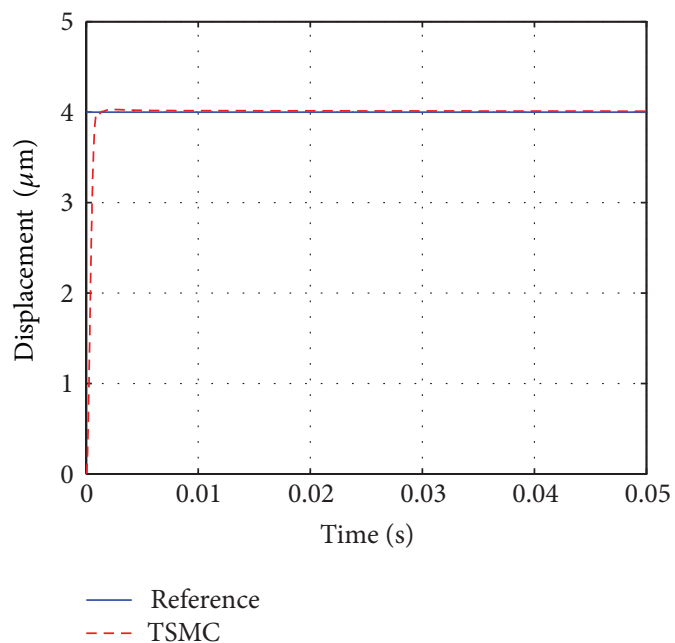

(d)

FIGURE 2: Simulation responses to step signals with amplitudes of (a) $1 \mu \mathrm{m}$, (b) $2 \mu \mathrm{m}$, (c) $3 \mu \mathrm{m}$, and (d) $4 \mu \mathrm{m}$.

TABle 1: Parameters of the PEA with Bouc-Wen model.

\begin{tabular}{lc}
\hline Parameter & Value \\
\hline$n$ & 1 \\
$\xi$ & $1.2315 \times 10^{4}$ \\
$\omega_{n}$ & $1.2225 \times 10^{6}$ \\
$k$ & $1.7339 \times 10^{-6}$ \\
$\alpha$ & 0.3575 \\
$\beta$ & 0.0364 \\
$\gamma$ & 0.0272 \\
\hline
\end{tabular}

4.2. Step Responses. The transient response capability of the proposed controller is examined firstly. The controller parameters for all simulations of this paper are shown in Table 2, and the results for steps of different amplitudes are described in Figure 2 and tabulated in Table 3 for a clear expression.

The simulation results observed from Figure 2 and Table 3 show that the proposed controller provides a smooth
TABle 2: Parameters of the implemented controller.

\begin{tabular}{lc}
\hline Parameter & Value \\
\hline$\mu$ & 1 \\
$\lambda$ & 0.85 \\
$\rho$ & 0.5 \\
$\tau$ & 0.01 \\
$k_{0}$ & $2.5 \times 10^{4}$ \\
$k_{1}$ & $1.5 \times 10^{7}$ \\
$k_{2}$ & $1.5 \times 10^{7}$ \\
$D+\epsilon$ & 5 \\
\hline
\end{tabular}

control with chattering free and fast convergence in finite time. Specifically, it can produce a fast response with a small overshoot.

4.3. Sinusoidal Tracking. The performances for tracking a sinusoidal waveform of $4 \mu \mathrm{m}$ peak-to-peak ( $\mathrm{p}$-p) amplitude 


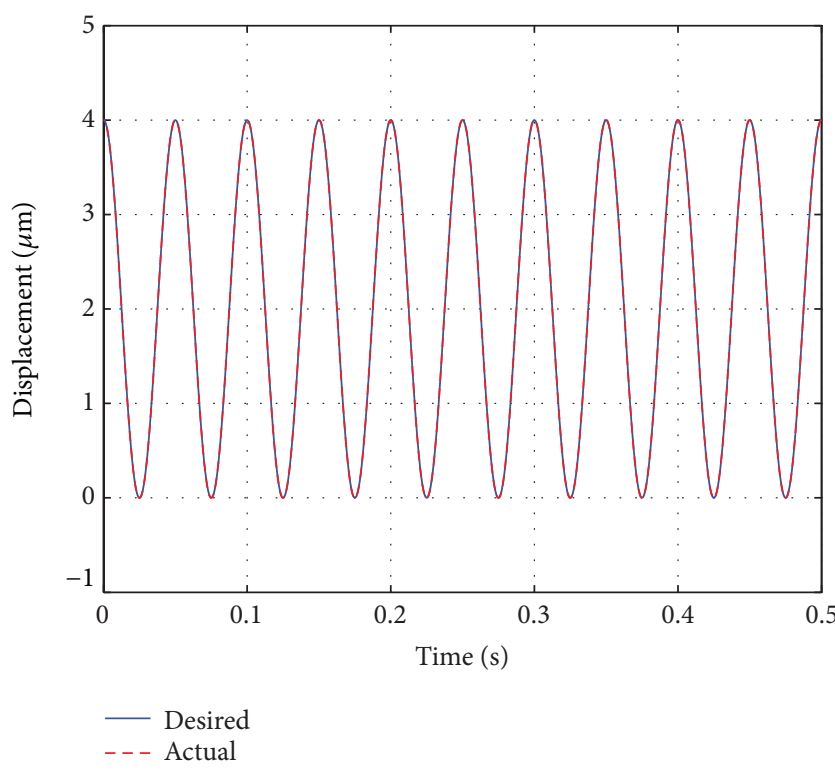

(a)

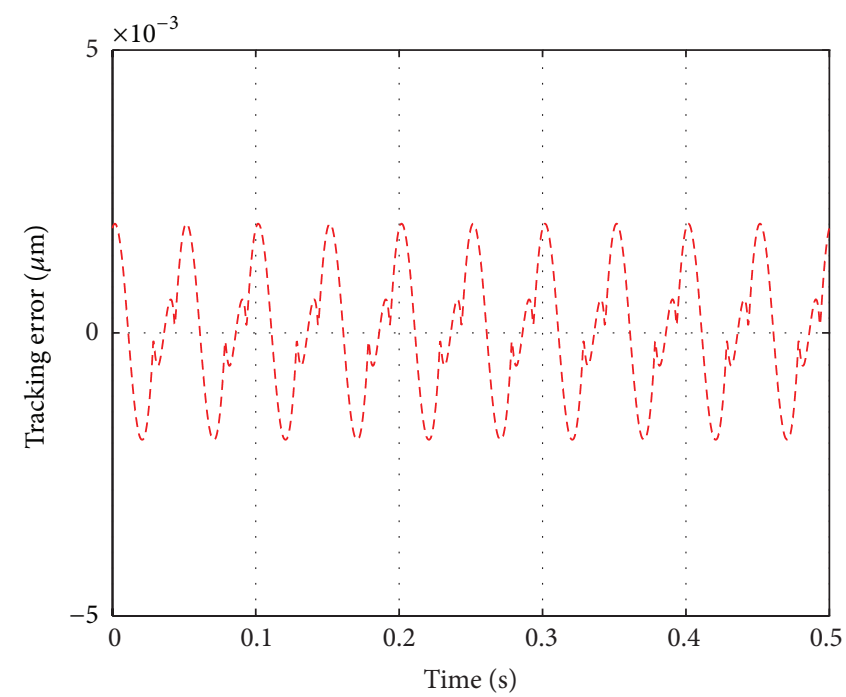

FIGURE 3: Simulation results of response to a $20 \mathrm{~Hz}$ sinusoidal signal.

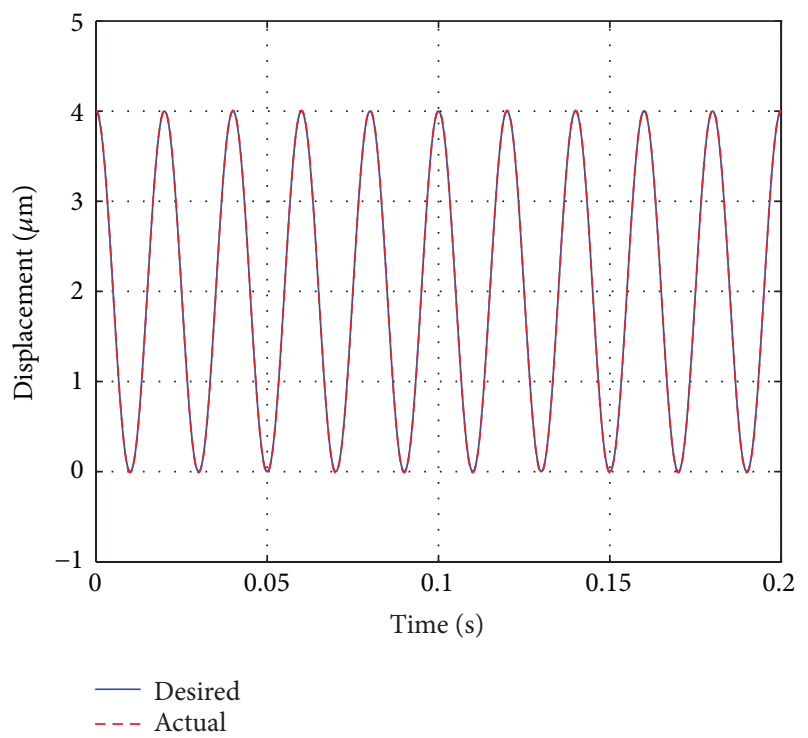

(a)

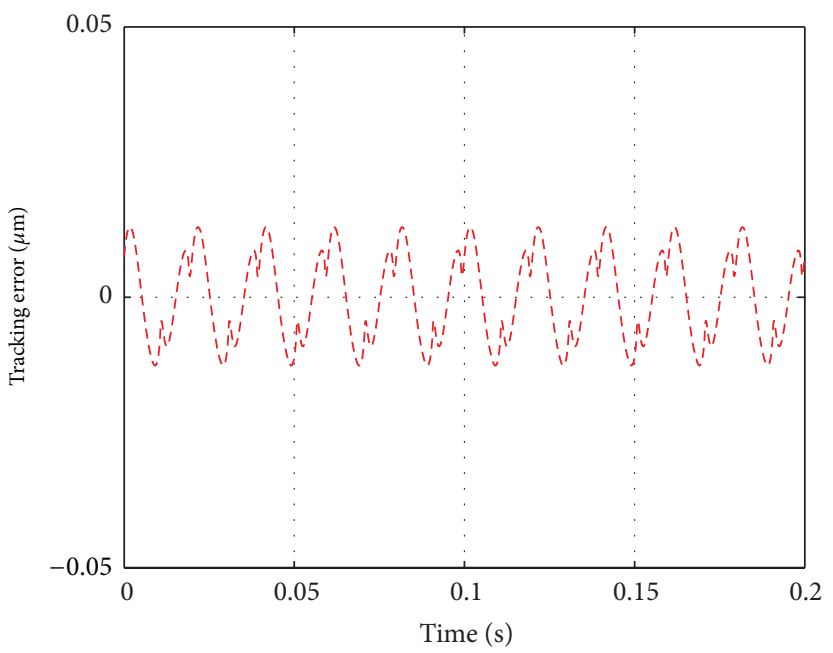

(b)

FIgURE 4: Simulation results of response to a $50 \mathrm{~Hz}$ sinusoidal signal.

TABLE 3: Control performance in step tracking.

\begin{tabular}{lcccc}
\hline $\begin{array}{l}\text { Performance } \\
\text { (with different amplitudes) }\end{array}$ & $1 \mu \mathrm{m}$ & $2 \mu \mathrm{m}$ & $3 \mu \mathrm{m}$ & $4 \mu \mathrm{m}$ \\
\hline 1\% settling time (ms) & 1.40 & 1.27 & 1.16 & 0.87 \\
Overshoot & $0.50 \%$ & $0.55 \%$ & $0.67 \%$ & $0.72 \%$ \\
\hline
\end{tabular}

in different frequencies using the proposed controller are depicted in Figures 3-4 and described in Table 4. It can be observed from the trajectories and tracking errors that the TSM controller can track the sinusoidal trajectory precisely and chattering free. It produces a maximum error of $\pm 0.0019 \mu \mathrm{m}$ at $20 \mathrm{~Hz}$ and $0.0130 \mu \mathrm{m}$ at $50 \mathrm{~Hz}$.

4.4. Responses to Staircase Signal. The staircase signal is applied to the proposed controller for the PEA. Figures 5(a) and 5(b) show that a step of the staircase signal covering the range of $1 \mu \mathrm{m}$ by 100 steps with each step lasting for $0.01 \mathrm{~s}$. The proposed controller can guarantee the steadystate error of $0 \mathrm{~nm}$ for approximating $80 \%$ duration of the step. Shorter distance positioning response is described in 


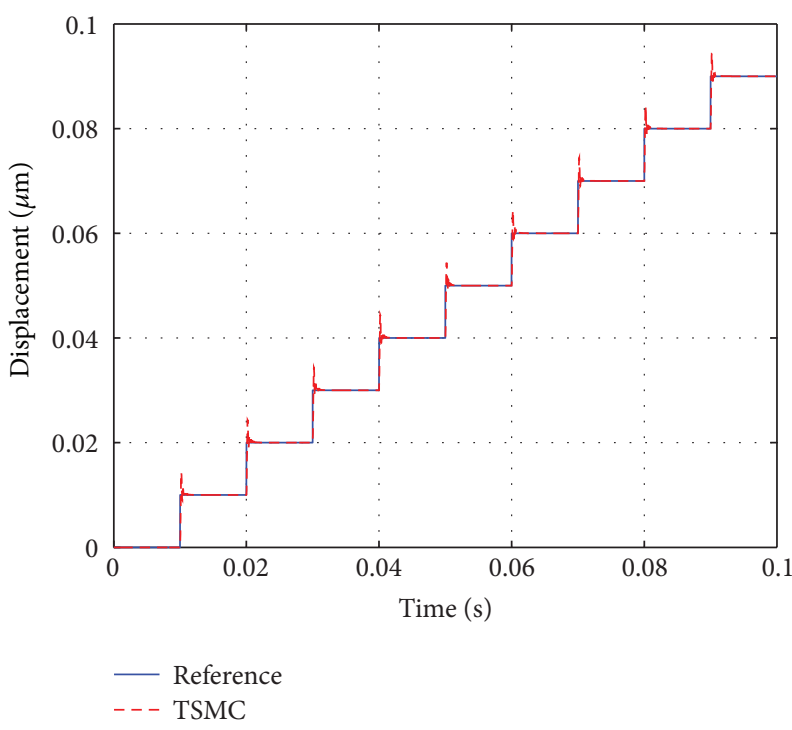

(a)

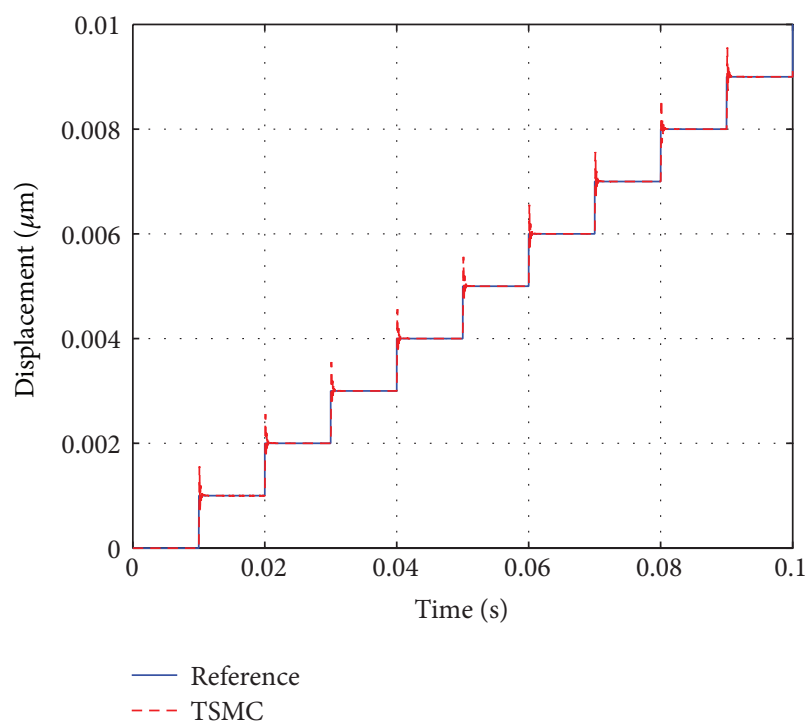

(c)

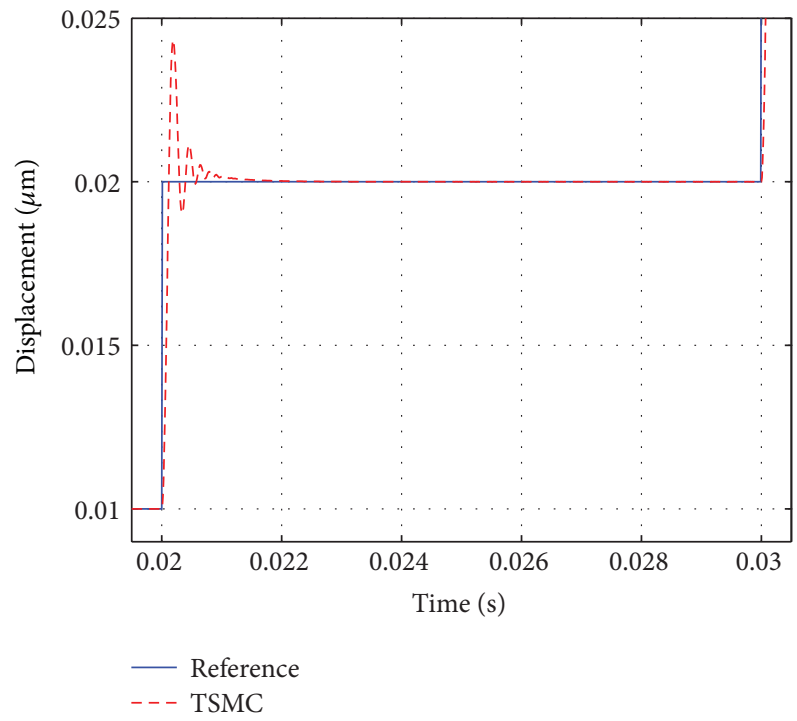

(b)

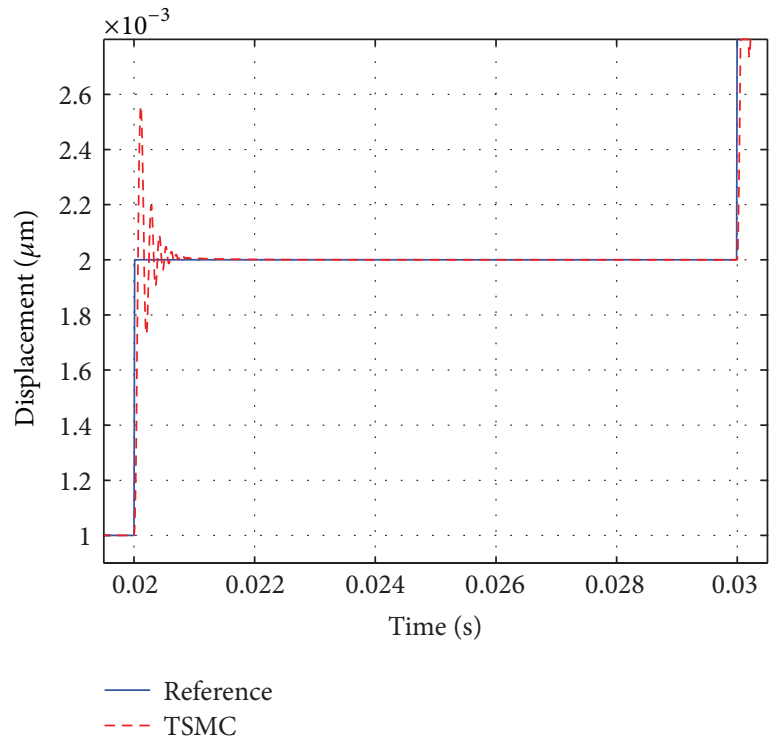

(d)

FIGURE 5: Simulation responses to staircase signals covering the range $1 \mu \mathrm{m}$ with (a)-(b) 100 steps and (c)-(d) 1000 steps.

TABLE 4: Performance of the controller with sinusoidal signal.

\begin{tabular}{lcc}
\hline $\begin{array}{l}\text { Performance } \\
\text { (with different frequencies) }\end{array}$ & RMSE $(\mu \mathrm{m})$ & Max. $E(\mu \mathrm{m})$ \\
\hline $5 \mathrm{HZ}$ & $1.3791 \times 10^{-8}$ & $-2.1021 \times 10^{-4}$ \\
$10 \mathrm{HZ}$ & $1.0575 \times 10^{-7}$ & $-5.0846 \times 10^{-4}$ \\
$20 \mathrm{HZ}$ & $1.4110 \times 10^{-6}$ & \pm 0.0019 \\
$50 \mathrm{HZ}$ & $6.8825 \times 10^{-5}$ & 0.0130 \\
$100 \mathrm{HZ}$ & 0.0012 & 0.0515 \\
\hline
\end{tabular}

Figures 5(c) and 5(d) in which the amplitude of each step is $1 \mathrm{~nm}$. The proposed controller can realize the steady-state error of $\pm 0.5 \mathrm{~nm}$ for approximating $85 \%$ duration of the step.
Therefore, the steps can be identified which indicates that the positioning resolution of the proposed controller is less than $1 \mathrm{~nm}$.

4.5. Discussions on Control Performance. In view of the simulation results, it can be concluded that the proposed TSM controller can obtain good performances in both positioning control and tracking control of the PEA. In the step signal simulations, the proposed controller enables a fast transient response without much overshoot, and especially, it removes the chattering without steady-state error. The TSM controller is also suitable for tracking control because of its small tracking error, fast response, and high resolution in both sinusoidal tracking and stair signals tracking. 


\section{Conclusions}

In this paper, a robust control strategy based on a new TSMC combined with a SMDO is developed for piezoelectric actuators. In order to get accurate motion tracking performance, the hysteresis model is considered in the PEA model for simulation. The step response simulation results show that the proposed controller can accelerate the transient response with low overshoot. In addition, it provides a smooth control and excellent performance in the control implementation yielding few chattering and fast convergence. The sinusoidal motion and stair signal tracking simulation results illustrate that the proposed controller can give a rise to the tracking performance with a small tracking error and high resolution. Based on this control strategy, the design of the controller is simple and convenient to drive the piezoelectric actuator. Robust stability of the proposed controller is guaranteed with the nonlinear uncertainties and external disturbance.

In the future research, fault detection and fault tolerant control of piezoelectric actuators will be an interesting work based on related results $[44,45]$.

\section{Conflict of Interests}

The authors declare that there is no conflict of interests regarding the publication of this paper.

\section{Acknowledgment}

The authors would like to acknowledge the financial support of the National Key Scientific Instrument and Equipment Development Projects of China through grant nos. 2012 YQ04014001 and 2012YQ04014010.

\section{References}

[1] S. Devasia, E. Eleftheriou, and S. O. R. Moheimani, "A survey of control issues in nanopositioning," IEEE Transactions on Control Systems Technology, vol. 15, no. 5, pp. 802-823, 2007.

[2] H. Song, G. Vdovin, R. Fraanje, G. Schitter, and M. Verhaegen, "Extracting hysteresis from nonlinear measurement of wavefront-sensorless adaptive optics system," Optics Letters, vol. 34, no. 1, pp. 61-63, 2009.

[3] D. Croft, G. Shedd, and S. Devasia, "Creep, hysteresis, and vibration compensation for piezoactuators: atomic force microscopy application," in Proceedings of the American Control Conference, vol. 3, pp. 2123-2128, Chicago, Ill, USA, June 2000.

[4] S. O. R. Moheimani, "Invited review article: accurate and fast nanopositioning with piezoelectric tube scanners: emerging trends and future challenges," Review of Scientific Instruments, vol. 79, no. 7, Article ID 071101, 2008.

[5] K. W. Chan and W.-H. Liao, "Precision positioning of hard disk drives using piezoelectric actuators with passive damping," in Proceedings of the IEEE International Conference on Mechatron$i c s$ and Automation (ICMA '06), pp. 1269-1274, Luoyang, China, June 2006.

[6] E. Eleftheriou, "Nanopositioning for storage applications," Annual Reviews in Control, vol. 36, no. 2, pp. 244-254, 2012.
[7] H. J. M. T. A. Adriaens, W. L. de Koning, and R. Banning, "Modeling piezoelectric actuators," IEEE/ASME Transactions on Mechatronics, vol. 5, no. 4, pp. 331-341, 2000.

[8] J. Li and L. Yang, "Adaptive pi-based sliding mode control for nanopositioning of piezoelectric actuators," Mathematical Problems in Engineering, vol. 2014, Article ID 357864, 10 pages, 2014.

[9] G. M. Clayton, S. Tien, A. J. Fleming, S. O. R. Moheimani, and S. Devasia, "Inverse-feedforward of charge-controlled piezopositioners," Mechatronics, vol. 18, no. 5-6, pp. 273-281, 2008.

[10] J. Minase, T.-F. Lu, B. Cazzolato, and S. Grainger, "A review, supported by experimental results, of voltage, charge and capacitor insertion method for driving piezoelectric actuators," Precision Engineering, vol. 34, no. 4, pp. 692-700, 2010.

[11] Y. T. Ma, L. Huang, Y. B. Liu, and Z. H. Feng, "Note: creep character of piezoelectric actuator under switched capacitor charge pump control," Review of Scientific Instruments, vol. 82, no. 4, Article ID 046106, 2011.

[12] K. K. Leang, Q. Zou, and S. Devasia, "Feedforward control of piezoactuators in atomic force microscope systems," IEEE Control Systems Magazine, vol. 29, no. 1, pp. 70-82, 2009.

[13] D. Pesotski, H. Janocha, and K. Kuhnen, "Adaptive compensation of hysteretic and creep non-linearities in solid-state actuators," Journal of Intelligent Material Systems and Structures, vol. 21, no. 14, pp. 1437-1446, 2010.

[14] B. Mokaberi and A. A. G. Requicha, "Compensation of scanner creep and hysteresis for AFM nanomanipulation," IEEE Transactions on Automation Science and Engineering, vol. 5, no. 2, pp. 197-206, 2008.

[15] M.-J. Jang, C.-L. Chen, and J.-R. Lee, "Modeling and control of a piezoelectric actuator driven system with asymmetric hysteresis," Journal of the Franklin Institute, vol. 346, no. 1, pp. 17-32, 2009.

[16] L. Liu, K. Tan, S. Chen, C. Teo, and T. Lee, "Discrete composite control of piezoelectric actuators for high-speed and precision scanning," IEEE Transactions on Industrial Informatics, vol. 9, no. 2, pp. 859-868, 2013.

[17] Y. C. Huang and D. Y. Lin, "Ultra-fine tracking control on piezoelectric actuated motion stage using piezoelectric hysteretic model," Asian Journal of Control, vol. 6, no. 2, pp. 208-216, 2004.

[18] T.-J. Yeh, H. Ruo-Feng, and L. Shin-Wen, "An integrated physical model that characterizes creep and hysteresis in piezoelectric actuators," Simulation Modelling Practice and Theory, vol. 16, no. 1, pp. 93-110, 2008.

[19] L. Juhász, J. Maas, and B. Borovac, "Parameter identification and hysteresis compensation of embedded piezoelectric stack actuators," Mechatronics, vol. 21, no. 1, pp. 329-338, 2011.

[20] H. G. Xu, T. Ono, and M. Esashi, "Precise motion control of a nanopositioning PZT microstage using integrated capacitive displacement sensors," Journal of Micromechanics and Microengineering, vol. 16, no. 12, article 2747, 2006.

[21] C.-Y. Lin and P.-Y. Chen, "Precision tracking control of a biaxial piezo stage using repetitive control and double-feedforward compensation," Mechatronics, vol. 21, no. 1, pp. 239-249, 2011.

[22] A. Sebastian and S. M. Salapaka, "Design methodologies for robust nano-positioning," IEEE Transactions on Control Systems Technology, vol. 13, no. 6, pp. 868-876, 2005.

[23] M. Boukhnifer and A. Ferreira, " $H_{\infty}$ loop shaping bilateral controller for a two-fingered tele-micromanipulation system," IEEE Transactions on Control Systems Technology, vol. 15, no. 5, pp. 891-905, 2007. 
[24] S. Yin, S. X. Ding, A. H. A. Sari, and H. Hao, "Data-driven monitoring for stochastic systems and its application on batch process," International Journal of Systems Science, vol. 44, no. 7, pp. 1366-1376, 2013.

[25] J. Dong, S. M. Salapaka, and P. M. Ferreira, "Robust control of a parallel-kinematic nanopositioner," Journal of Dynamic Systems, Measurement and Control, vol. 130, no. 4, Article ID 0410071, 15 pages, 2008.

[26] T. W. Seo, H. S. Kim, D. S. Kang, and J. Kim, "Gain-scheduled robust control of a novel 3-DOF micro parallel positioning platform via a dual stage servo system," Mechatronics, vol. 18, no. 9, pp. 495-505, 2008.

[27] H. C. Liaw, B. Shirinzadeh, and J. Smith, "Enhanced sliding mode motion tracking control of piezoelectric actuators," Sensors and Actuators A, vol. 138, no. 1, pp. 194-202, 2007.

[28] H. C. Liaw, B. Shirinzadeh, and J. Smith, "Sliding-mode enhanced adaptive motion tracking control of piezoelectric actuation systems for micro/nano manipulation," IEEE Transactions on Control Systems Technology, vol. 16, no. 4, pp. 826-833, 2008.

[29] J.-C. Shen, W.-Y. Jywe, C.-H. Liu, Y.-T. Jian, and J. Yang, "Sliding-mode control of a three-degrees-of-freedom nanopositioner," Asian Journal of Control, vol. 10, no. 3, pp. 267-276, 2008.

[30] G. Song, J. Zhao, X. Zhou, and J. A. de Abreu-García, "Tracking control of a piezoceramic actuator with hysteresis compensation using inverse Preisach model," IEEE/ASME Transactions on Mechatronics, vol. 10, no. 2, pp. 198-209, 2005.

[31] C.-J. Lin and S.-R. Yang, "Precise positioning of piezo-actuated stages using hysteresis-observer based control," Mechatronics, vol. 16, no. 7, pp. 417-426, 2006.

[32] O. Barambones and V. Etxebarria, "Robust sliding composite adaptive control for mechanical manipulators with finite error convergence time," International Journal of Systems Science, vol. 32, no. 9, pp. 1101-1108, 2001.

[33] O. Barambones and V. Etxebarria, "Energy-based approach to sliding composite adaptive control for rigid robots with finite error convergence time," International Journal of Control, vol. 75, no. 5, pp. 352-359, 2002.

[34] S. Yin, G. Wang, and H. R. Karimi, "Data-driven design of robust fault detection system for wind turbines," Mechatronics, 2013.

[35] Y. Feng, X. Yu, and Z. Man, "Non-singular terminal sliding mode control of rigid manipulators," Automatica, vol. 38, no. 12, pp. 2159-2167, 2002.

[36] S. Yu, X. Yu, B. Shirinzadeh, and Z. Man, "Continuous finitetime control for robotic manipulators with terminal sliding mode," Automatica, vol. 41, no. 11, pp. 1957-1964, 2005.

[37] J. Y. Peng and X. B. Chen, "Integrated pid-based sliding mode state estimation and control for piezoelectric actuators," IEEE/ASME Transactions on Mechatronics, vol. 19, no. 1, pp. 8899, 2012.

[38] V. T. Haimo, "Finite time controllers," SIAM Journal on Control and Optimization, vol. 24, no. 4, pp. 760-770, 1986.

[39] Y. Hong, J. Huang, and Y. Xu, "On an output feedback finitetime stabilization problem," IEEE Transactions on Automatic Control, vol. 46, no. 2, pp. 305-309, 2001.

[40] D. Zhao, S. Li, and F. Gao, "A new terminal sliding mode control for robotic manipulators," International Journal of Control, vol. 82, no. 10, pp. 1804-1813, 2009.
[41] C. E. Hall and Y. B. Shtessel, "Sliding mode disturbance observer-based control for a reusable launch vehicle," Journal of Guidance, Control, and Dynamics, vol. 29, no. 6, pp. 1315-1328, 2006.

[42] S. Yin, X. Yang, and H. R. Karimi, "Data-driven adaptive observer for fault diagnosis," Mathematical Problems in Engineering, vol. 2012, Article ID 832836, 21 pages, 2012.

[43] T. S. Low and W. Guo, "Modeling of a three-layer piezoelectric bimorph beam with hysteresis," Journal of Microelectromechanical Systems, vol. 4, no. 4, pp. 230-237, 1995.

[44] S. Yin, H. Luo, and S. Ding, "Real-time implementation of faulttolerant control systems with performance optimization," IEEE Transactions on Industrial Electronics, vol. 61, no. 5, pp. $2402-$ 2411, 2014.

[45] S. Yin, S. Ding, A. Haghani, H. Hao, and P. Zhang, "A comparison study of basic data-driven fault diagnosis and process monitoring methods on the benchmark tennessee eastman process," Journal of Process Control, vol. 22, no. 9, pp. 1567-1581, 2012. 


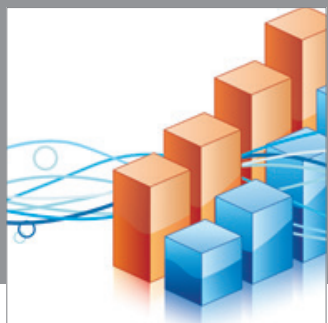

Advances in

Operations Research

mansans

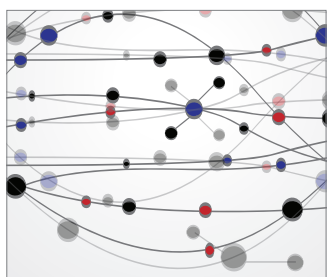

The Scientific World Journal
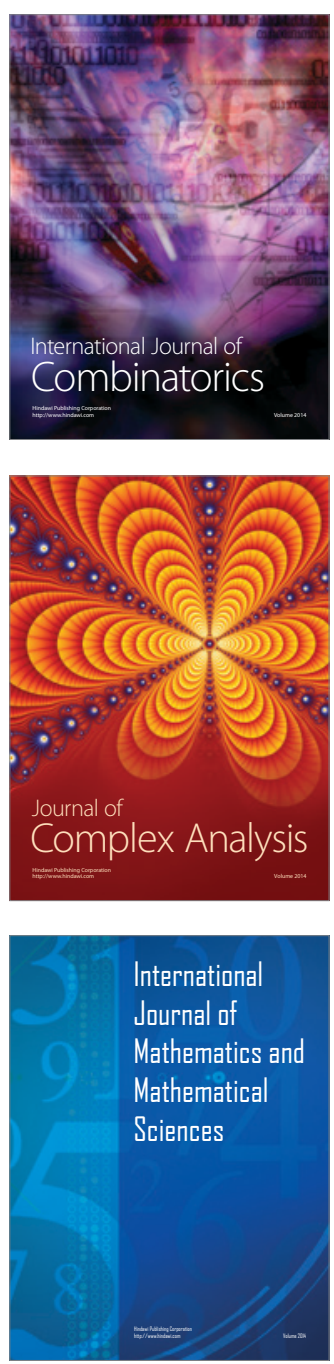
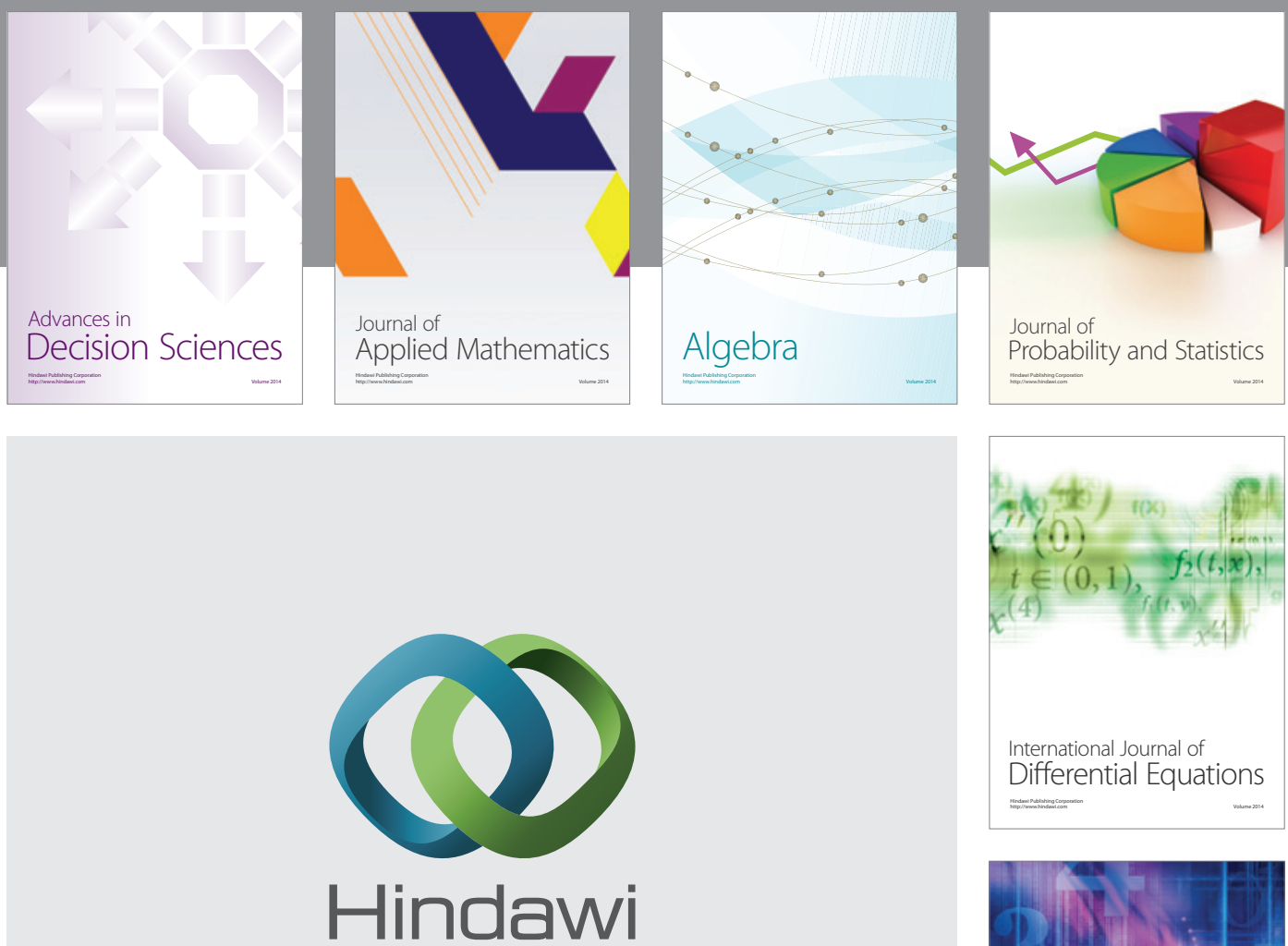

Submit your manuscripts at http://www.hindawi.com
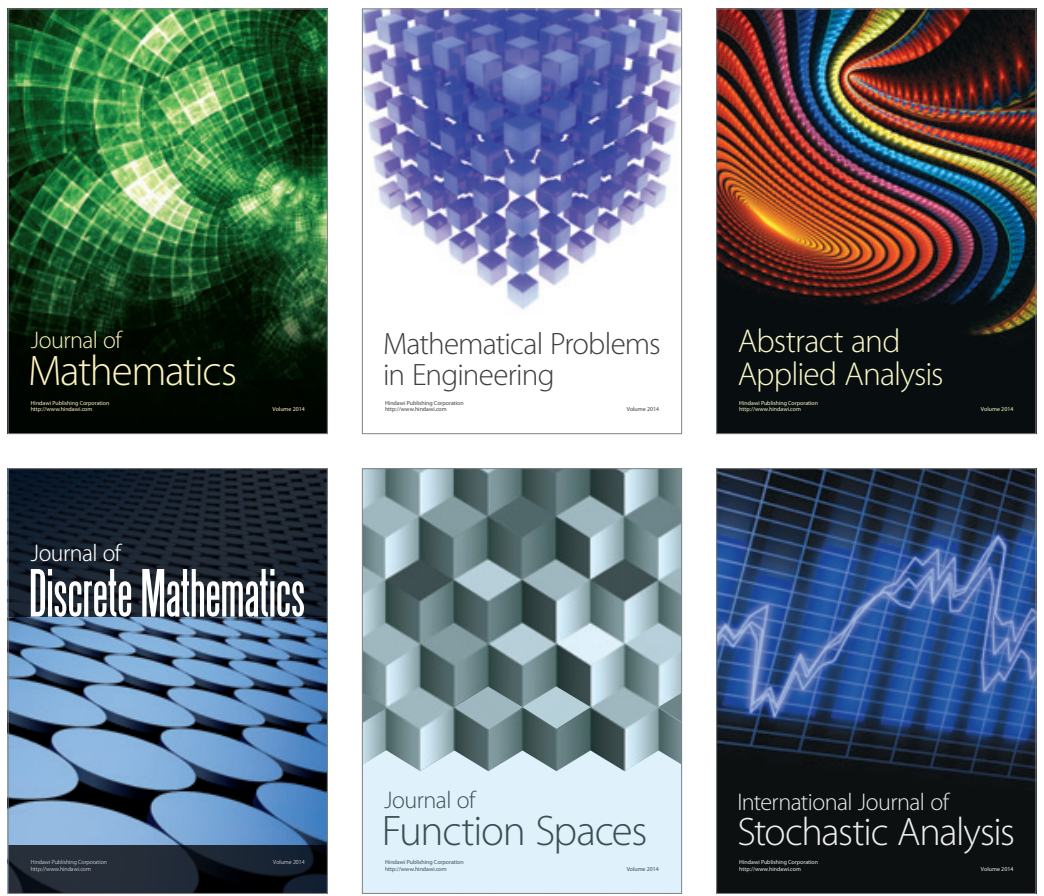

Journal of

Function Spaces

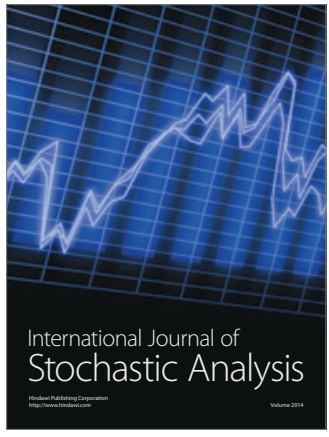

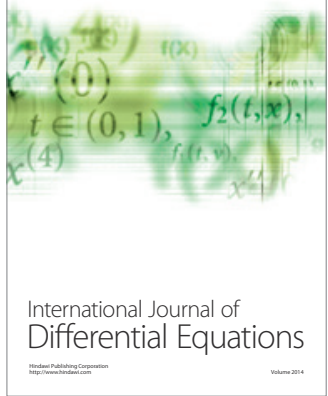
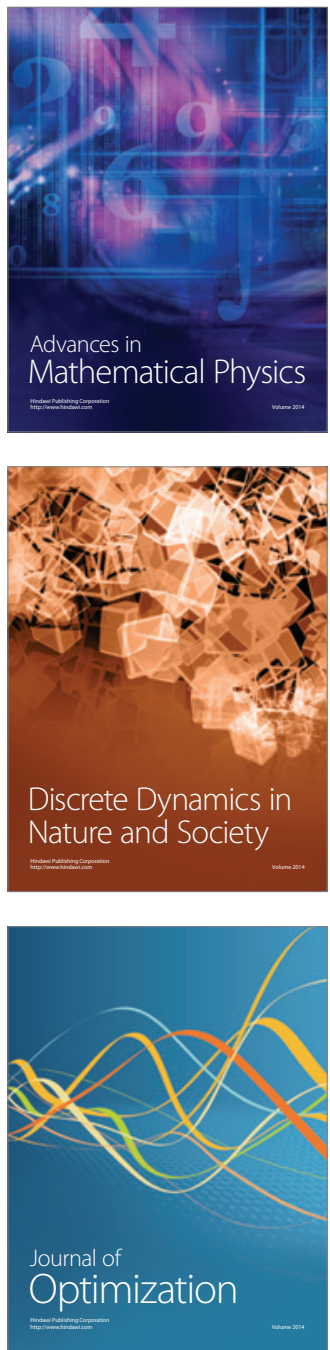